\title{
De l'autorité ethnographique à l'autorité du corps et des émotions
}

\author{
Réflexions à partir d'un terrain "chez soi" \\ auprès d'une minorité, les "roms " en Italie
}

\begin{abstract}
Alice Sophie Sarcinelli ${ }^{1}$
[Résumé] Sur base d'une recherche sur l'enfance et la parentalité roms en Italie, menée «chez soi » mais auprès d'une minorité, l'article interroge l'engagement émotionnel et corporel de l'ethnographe pour saisir la portée de démarches réflexives et « réflectives » (Csordas, 1999) sous les angles théorique, méthodologique et épistémologique. Après examen des critiques faites aux théories postmodernes et considérant le savoir ethnographique comme le produit d'une expérience incarnée fondée sur la rencontre entre le chercheur et ses interlocuteurs, je proposerai dans un premier temps l'emploi de la réflectivité comme outil complémentaire à la réflexivité. Dans un deuxième temps, j'analyserai les émotions suscitées dans cette rencontre, décrivant ses dimensions objectives - mes caractéristiques sociales et celles de mes interlocuteurs - et subjectives - mes émotions et l'expérience interactionnelle des enquêtés. Je montrerai enfin que si les données objectives permettent d'examiner la relation d'enquête en tant que rapport social de sexe, d'âge et de statut, le focus sur I'(inter)subjectivité contribue à ce même travail d'objectivation des rapports dans l'interaction.
\end{abstract}

Mots-clés : autorité ethnographique, émotions, ethnographie chez soi, Roms, réflexivité.

[Abstract] Based on an ethnographic research on Rom childhood and parenthood in Italy, conducted «at home » but with a social minority, this paper questions the ethnographer's emotional and bodily engagement to discuss the importance of reflexivity and reflectivity (Csordas, 1999) from theoretical, methodological and epistemological points of view. In the first part I examine postmodernism critics and alternatives. Arguing that ethnographic knowledge results from embodied experience within the ethnographic encounters and relations, I propose reflectivity as an analytical tool complementary to reflexivity. In the second part, I analyse the emotions emerging from such an encounter and I describe its objective and subjective dimensions (my social characteristics and those of my interlocutors as well as my emotions and the interactional experience of my interlocutors). I finally show that while objective data allows to examine the ethnographic relationship as social sex, age and status bonds, the focus on (inter)subjectivity contributes to objectivizing these bonds within interactions.

Keywords: ethnographic authority, emotions, fieldwork at home, Roms, reflexivity.

${ }^{1}$ FRS-FNRS/Université de Liège. 


\section{Introduction}

Par rapport à mes précédentes enquêtes au Brésil (Sarcinelli, 2007), mon travail de thèse sur l'enfance et de la parentalité « roms $»^{2}$ en Italie (Sarcinelli, 2014), réorientait mes recherches depuis l'anthropologie du loin vers l'anthropologie du proche. J'entamais aussi une recherche "chez moi » (Peirano, 1998) auprès d'une minorité3 stigmatisée. Circulant entre des mondes sociaux familiers (le réseau associatif et militant) et d'autres qui m'étaient totalement inconnus (les campements roms), j'ai été perçue différemment selon les milieux, me rapprochant de la figure de la «native multiple » (Mascarenhas-Keyes, 1987). Souvent je n'étais pas considérée comme « tout à fait native » (Peirano, 1998: 109) en raison des années passées à l'étranger et de mon affiliation à une institution française. Cette position me donnait dans bien des cas plus de légitimité, surtout vis-à-vis des militants de petits groupes informels qui se sentaient valorisés par le fait qu'une enquêtrice venant de l'étranger s'intéresse à leur expérience. En revanche, le terrain auprès de familles roms a été caractérisé par la complexité de la rencontre avec une minorité stigmatisée (Bordigoni, 2001; Okely, 1984), ainsi que par la présence d'activités illégales et criminelles, à différents degrés, chez une partie de mes interlocuteurs. Peu importait si j'étais travailleuse sociale, institutrice, bénévole, journaliste ou chercheuse, j'étais avant tout une Gadjí (femme non-rom) et, de surcroît, une représentante de la société dominante.

Afin de rendre intelligibles les données empiriques issues de ce travail, il m'est apparu comme une condition sine qua non de problématiser les rapports sociaux et les registres relationnels pesant sur l'enquête de terrain menée "chez soi», bien qu'auprès d'une minorité. J'utiliserai comme données mes propres caractéristiques sociales et celles de mes interlocuteurs, ainsi que la dimension émotionnelle des interactions d'enquête. Ce cas spécifique est pris à titre d'exemple pour réfléchir sur l'utilité et le sens des démarches réflexives et "réflectives », comme on l'expliquera au fil du texte. J'examinerai d'abord l'utilité et le sens des démarches réflexives pour en-

\footnotetext{
${ }^{2}$ L'usage des guillemets permet de souligner le processus de catégorisation dont ces populations font l'objet. Par convention, j'ai choisi de ne pas le poursuivre dans la suite du texte. ${ }^{3}$ Je ne me réfère pas ici au concept de minorité ethnique mis en avant par de nombreux organismes internationaux qui considèrent les Roms comme la principale minorité ethnique de l'Union européenne, mais à l'acception proposée par Didier et Éric Fassin (2006) comme «catégorie naturalisée par la discrimination » (p. 251). J'ai donc employé le terme de «minorité » pour me référer à l'ensemble des groupes et des personnes qui sont discriminés par les pouvoirs publics ou dans le monde social du fait d'être identifiés comme roms ou tsiganes.

${ }^{4}$ Pour ce mot, j'ai gardé l'orthographe phonétique Gadjo, le pluriel Gadje et le féminin Gadji qui apparaissent dans les écrits des auteurs francophones.
} 
suite restituer les spécificités et les contraintes de l'enquête, ainsi que la dimension émotionnelle des interactions avec mes interlocuteurs.

\section{De l'autorité ethnographique à l'autorité des émotions}

L'analyse de la relation ethnographique soulève la question du processus de production du savoir, un débat introduit par l'anthropologie anglophone qui n'a pas été sans écho dans le monde francophone (Cefaï, 2010). La théorie postmoderne (Clifford, Marcus, 1986) a interrogé l'écriture, comme ensemble des stratégies rhétoriques vouées à convaincre le lecteur de la « vérité » que l'on présente, ainsi que les processus de formation et de remise en question de l'autorité de l'ethnographe (Clifford, 1996). L'apport de cette théorie est, certes, indéniable, mais des critiques de plusieurs ordres lui ont été adressées. Primo, Bourgois (2001) avance une critique d'ordre politique en reprochant la tendance élitiste à s'enfermer dans un discours universitaire et à nier l'expérience personnelle. Une deuxième, d'ordre épistémologique, remet en question la valeur heuristique de cette approche (Okely, 1992) : certains auteurs ont cherché à mener en ce sens de véritables analyses critiques de la situation ethnographique (Fassin, Bensa, 2008; Pack, 2006) pour en découvrir les enjeux à la fois épistémologiques, éthiques et politiques. Une troisième critique puise ses origines dans l'anthropologie du corps (Faavret-Saada, Contreras, 1981). Plus particulièrement, les théories de l'incorporation issues de la phénoménologie culturelle reprochent à la critique réflexive de rester complètement textuelle, ne parvenant pas à offrir une véritable alternative au primat des représentations. Edward Bruner (1986) déplore la tendance à se focaliser sur la verbalisation au détriment de la visualisation, tendance qui empêche d'interroger simultanément l'expression et l'incorporation de l'expérience. En contrepoint, Thomas J. Csordas (1999) propose une anthropologie à partir du corps, analysant la dimension corporelle du processus d'acculturation propre à l'enquête ethnographique. Il pose la « réflectivité » comme outil complémentaire à la réflexivité et présente le savoir comme le produit d'une expérience incarnée des chercheurs qui utilisent leur propre corps comme instrument de recherche et comme lieu d'un processus mimétique. L'enquête n'est pas uniquement la somme de dialogues et de conversations, mais vise l'ensemble des expériences corporelles et sensorielles qui jouent un rôle fondamental dans la production des représentations ethnographiques. D'autres auteurs se sont intéressés aux processus corporels de perception: l'expérience ethnographique est entendue comme pratique intersubjective (Turner, 2000) où l'ethnographe participe à la vie quotidienne avec son propre corps (Jackson, 1983). Ce travail d'analyse de la dimension corporelle de la rencontre ethnographique permet à la fois d'avoir un aperçu sur l'expérience de l'autre et de réfléchir au corps de l'ethnographe comme centre propulseur de l'élaboration du savoir. Si l'expérience incorporée du chercheur est une donnée fondamentale pour situer le produit anthro- 
pologique, elle est peut-être complétée par l'apport, voire l'autorité des émotions, ou mieux des corps traversés par l'émotionnel (Scheper-Hughes, 1992).

\section{Une ethnographie dans un camp « non autorisé mais toléré »}

La question de l'enfance et de la parentalité rom en Italie a été explorée, d'une part à travers une ethnographie de la vie quotidienne dans des campements roms de la région de Milan et d'autre part une ethnographie de l'espace public et des politiques locales, nationales et européennes. Je me concentrerai ici sur l'ethnographie menée au sein des campements roms de Milan. J'entretenais avec la ville où j’ai grandi « un lien de localité, fondé sur une connaissance en termes de lieux, pas sur une connaissance de l'objet d'étude" » (Weber, Noiriel, 1990 : 140). Cela m’a permis de développer des réseaux plutôt rapidement, par des voies informelles. Faute de liens préalables, je suis entrée en contact avec les familles roms à travers des intermédiaires, consciente du risque de me retrouver mêlée aux rapports souvent complexes entre habitants des campements roms et acteurs institutionnels ou associatifs. Cela dit, l'expérience a été très différente selon les contextes : dans les paragraphes qui viennent je me concentrerai sur deux volets de l'enquête, réalisés l'un dans un campement illégal et toléré, et l'autre auprès des familles se déplaçant de bidonville en bidonville au rythme des expulsions.

Un premier terrain s'est ouvert au campo non autorisé de Cainazate - défini par les pouvoirs publics comme illégal et toléré ${ }^{6}$ - où j'ai été accompagnée la première fois par M. Ferrari, leader local d'une organisation historiquement engagée dans la défense des minorités roms. Contrairement à mes idées préconçues - influencées en partie par les activités d'insertion de l'organisation - je me suis retrouvée dans un quartier plein de voitures coûteuses et de maisons bien entretenues, remplies d'objets de luxe et de technologie, très différents d'autres campements où j'avais tenté mon immersion (Sarcinelli, 2014).

\footnotetext{
${ }^{5}$ J'ai utilisé des pseudonymes et changé certaines caractéristiques sociales de mes interlocuteurs, de manière à garantir leur anonymat et la confidentialité.

${ }^{6}$ Le terme « campi rom (camps roms) » prête à malentendu (Piasere, 2006). Dans le langage commun, il est utilisé pour nommer des emplacements qui ont des statuts très différents : des camps municipaux autorisés, des bidonvilles non autorisés et beaucoup d'autres lieux habités par des Roms ayant des statuts plus ambigus, qui sont généralement connus sous le terme de "camps tolérés ». Le terme « camp non autorisé mais toléré » est celui utilisé par la police locale dans son document interne de classification des campements roms présents sur le territoire. Au fil du texte, j'ai pris la liberté d'utiliser le terme camp, campement ou terrain pour des questions de lisibilité.
} 


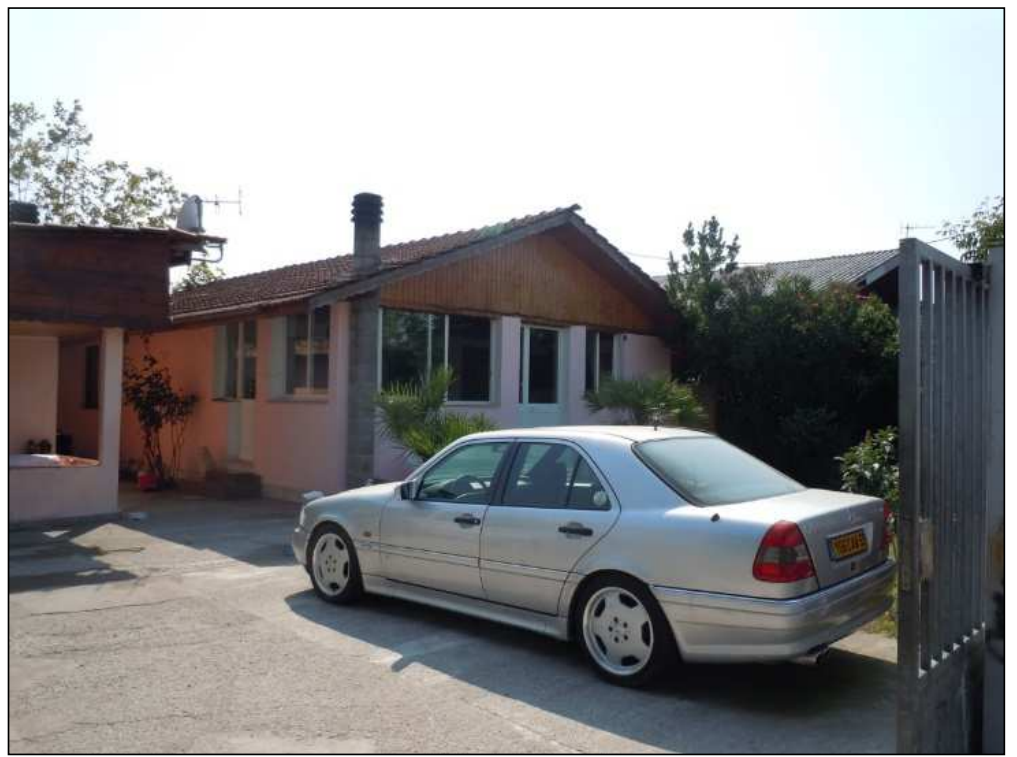

Figure $n^{\circ} 1$. Une maison du campo Monte Bisbino [cliché : Sarcinelli]

La relative stabilité des familles rend ici mon insertion sur le terrain plus facile, quoique la présence d'activités illégales ne manquera pas de me poser des questions éthiques tout au long de la thèse, de la recherche sur le terrain jusqu'à la phase d'analyse, d'écriture et de restitution (Sarcinelli, 2014).

Bien que M. Ferrari me présente aux habitants comme une étudiante et que je reviendrai par la suite toute seule pour fréquenter les familles, elles me prennent d'abord pour une bénévole ou pour une maîtresse de M. Ferrari. Je multiplie alors mes stratégies pour ne pas être associée à lui et rapidement j’arrive à développer des relations d'enquête de façon autonome. J'insiste sur mon travail de recherche, soulignant les aspects plus positifs de leur identité (les cultures enfantines et éducatives) et minimisant leur réputation (assez répandue) de parents défaillants. Néanmoins, dans cette première phase, je me sens mal à l'aise de m’immiscer dans la vie privée de ces familles et j'éprouve des sentiments de peur et de dégoût vis-à-vis de ce lieu :

Je suis en train d'aller au camp toute seule. Je suis tendue. J'ai beaucoup attendu avant de sortir. Peut-être que je me sens sans défense ? Je veux arriver à un horaire qui me permet d'éviter de partager le repas avec eux, car ça me met mal à l'aise. Le lieu me paraît difficile à rejoindre... je me rapproche avec les transports en commun, mais ensuite j'ai peur de faire le sentier à pied. Aujourd'hui j'ai rencontré un homme en arrivant au sentier : j'ai eu peur de me retrouver toute seule face à un homme sur ce sentier-là... Puis, j'ai parcouru le sentier, ça ne prend que quelques minutes, mais ça me dégoûte : ça pue, il est plein de déchets... mais je n'ai pas d'alternatives. Arrivée devant la maison de Madame B. Slagena, j'avais la nausée (Cahier de terrain, 21/05/09). 
Je cherche à rentrer dans l'intimité familiale de la manière la plus discrète et la moins envahissante possible: dans l'impossibilité de fixer des rendez-vous précis, j'établis une routine me permettant d'en avoir sans en prendre. Après avoir passé d'interminables après-midis à jouer à cache-cache avec les enfants sous le soleil brûlant, un lien de proximité s'établit entre nous. En revanche, la suspicion des adultes demeure : sans rôle identifiable, ma présence demeure incompréhensible et peu justifiée, au minimum suspecte, voire résolument ambiguë. Mes interlocuteurs me croient soit idiote (car j'aurais pu mieux gagner ma vie en faisant autre chose), soit très riche, ou un imposteur qui cache quelque chose. Chacun se fait une idée sur mon identité (travailleuse sociale, institutrice, policière ou encore juge) comme c'est le cas de M. Tencovic:

Monsieur Tencovic: Mais qui t'oblige à venir tous les jours ici avec les Tsiganes? Es-tu italienne? Tu n'es pas italienne? Tu es sinta? Pourquoi tu viens ici ? Tu travailles? Ton père a beaucoup d'argent? Qu'est-ce que tu feras de ce travail ? Tu vas devenir juge ? [...]. Tu parles beaucoup de langues, alors tu es des services secrets! Tu dis que tu fais des études, mais qu'est-ce que tu vas faire après ? Juge ? [...]

Mme Tencovic: Et qu'est-ce que tu vas dire de nos enfants? Qu'ils sont méchants car ils ne vont pas à l'école ? [...] Vas-y! Prends un café avec nous !

M. T. : En tout cas je ne vais pas faire d'entretien. Et si je le fais, tu me donnes quelque chose ? Et toi, ça te rapporte quoi de faire ça?

Mon intégration progresse lorsque Marisa, institutrice à l'école du quartier, m'introduit auprès d'autres familles qu'elle fréquente depuis quelques années, dont les Brenovic. C'est une famille assez aisée : le père, concessionnaire, possède des papiers et sa famille est en cours de régularisation; les enfants sont tous nés à Milan et scolarisés depuis la maternelle, et la mère souhaite me payer pour leur donner des cours particuliers - ce que je refuse, afin d'entretenir des relations paritaires avec plusieurs familles pour mener à bien l'enquête. En raison de leur position sur le terrain et du lien que je tisse avec eux, j'acquière progressivement une légitimité et me sens protégée. En contrepartie, ils prétendent contrôler mes relations avec les autres habitants : je me retrouve prise dans des réseaux de complicité et d'inimitié auxquels je vais devoir me confronter pour le reste de l'enquête.

\section{Une ethnographie itinérante}

Mon intégration sur le deuxième terrain, par contre, a été bien plus longue et complexe, en dépit de l'intermédiation de militantes et de comités de soutien. Tout à fait différent est le contexte de vie de familles roms roumaines d'immigration récente qui, du fait d'évacuations répétées, se déplacent constamment, reconstruisant au fur et à mesure des bidonvilles à Milan et dans sa banlieue. La situation est particulièrement grave à cette période hivernale de l'année 2009 car la mairie s'acharne à multiplier les expulsions et la destruction des baraques et des biens, en laissant les familles à la rue (Sarcinelli, 2016). 


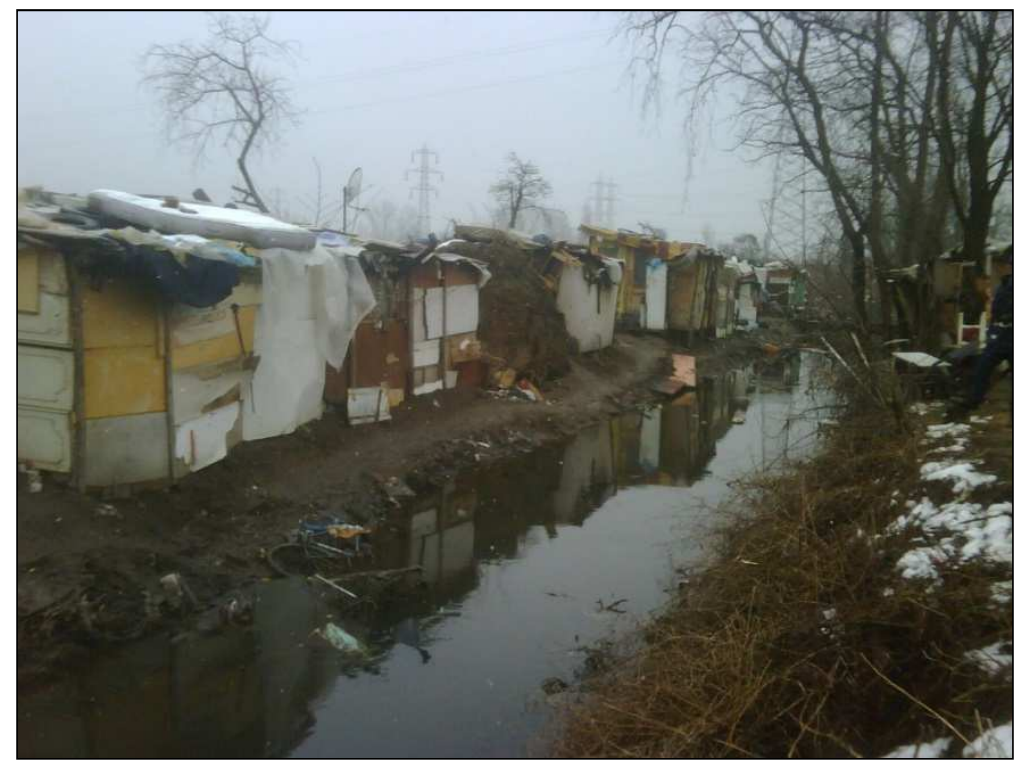

Figure $\mathrm{n}^{\circ}$ 2. Un bidonville rom [cliché : archives de Stefano Pasta]

La difficulté d'éviter les relations-type instaurées d'habitude avec les militants, les policiers, les acteurs de l'humanitaire, les travailleurs sociaux et les journalistes est majeure. Les relations d'aide et de solidarité reposant sur la compassion, ou bien celles de contrôle et de rejet basées sur des mesures répressives semblent les seuls types de relations possibles - la frontière entre un type de relation et l'autre étant souvent bien moins nette qu'on ne le penserait a priori. À cela s'ajoutent mes propres réactions émotionnelles et sentiments moraux : ces lieux insalubres, isolés, peuplés d'hommes ivres et de personnes extrêmement précaires m'accablent. Après de nombreuses tentatives, une véritable relation d'enquête s'établit enfin avec les Gheorghe, une famille composée par un couple, leurs quatre enfants mineurs, leur belle-fille d'une vingtaine d'années et ses deux petits. Puisque les expulsions m'empêchent de mener une ethnographie géographiquement située, je commence une ethnographie itinérante, suivant la famille dans ses parcours entre bidonvilles, centres d'hébergement, la rue et « ses » lieux de vie (écoles, structures sanitaires, etc.). Dans les semaines qui suivent, la bellefille Gheorghe me demande d'être la marraine à son baptême et à celui de ses enfants. Je participe donc à la préparation matérielle et organisationnelle du baptême7 ${ }^{7}$.'est ainsi que j'acquière des droits et des obligations : je me dois de jouer le rôle de média-

\footnotetext{
${ }^{7}$ Les baptêmes ont été réalisés par un prêtre catholique, alors que la famille est d'origine orthodoxe.
} 
trice (avec l'école, les intervenants sociaux, le personnel hospitalier et les militants) et j'ai «droit » à «ma » partie des jupes récupérées et des fruits offerts par les enseignants. À la différence des autres non-roms ${ }^{8}$ en contact avec la famille, je suis la seule à franchir des frontières symboliques, comme le fait d'aller avec eux en voiture, de partager des repas ou de les assister pendant qu'ils « récupèrent » des vêtements dans la benne de Caritas. Cette intimité a pourtant été à l'origine d'un "contrat incomplet »" tout en ayant clarifié les raisons de ma présence à plusieurs reprises, la famille a continué à me considérer comme leur marraine tout au long de l'enquête, laissant de côté mon statut d'enquêtrice.

\section{L'intégration du corps ambigu de l'anthropologue}

Dans les deux terrains, c'est avec un corps dont le statut est ambigu que je rentre en relation avec mes interlocuteurs. Dans les réseaux des familles élargies qui ont fait l'objet de cette enquête, la distinction entre l'enfance et l'âge adulte se fait pour une femme en fonction du passage du statut de vierge/célibataire à celui de femme/déflorée (Sarcinelli, 2015). Célibataire, plutôt jeune, mais soupçonnée d'être rentrée dans la vie sexuelle active, mon statut est à mi-chemin entre celui de fille et de femme. Après mon propre mariage, survenu durant la deuxième partie du terrain, Sonia, l'une des jeunes filles de 13 ans (vierge et célibataire) dont j'étais la plus proche, m'a accusée de ne plus être la même. Or ce n'était pas tant moi qui avais changé vis-àvis de mes interlocuteurs que mon statut de leur point de vue, puisque le mariage constitue le passage à l'âge adulte. Au fil de l'enquête j'essaie donc de prendre conscience de ce corps au statut ambigu et de l'adapter pour établir différentes relations d'enquête. Pour m'intégrer au corps social des enfants Vlancovic (un groupe de cousins vivant au sein de la famille élargie), j'investis les espaces et les activités comme ils

\footnotetext{
${ }^{8}$ Pour distinguer entre les personnes appartenant aux minorités tsiganes et les autres, il est d'usage chez les chercheurs spécialisés sur ces populations d'adopter le terme Gadjo. Puisque ce genre de réappropriation d'une catégorie indigène me semble scientifiquement problématique, il me paraît préférable d'utiliser ce terme uniquement lorsque je reporte le point de vue des Roms et d'employer dans les autres cas le terme « non-Rom », beaucoup moins élégant, mais scientifiquement plus approprié.

${ }^{9}$ « La relation d'enquête semble entourée du même type d'indétermination, même après la présentation par l'enquêteur du projet d'investigation et la formulation d'un accord de la part de l'enquêté, même dans les contextes où est prévu un accord écrit, contresigné par les parties, sur la base d'une présentation précise et détaillée comme cela se pratique aux États-Unis. Quand ils donnent leur accord, les enquêtés savent-ils à quoi ils s'engagent? S'ils ne savent pas toujours bien ce qui les attend, ils s'en sont fait une idée : ils ont complété à leur façon le contrat » (Fournier, 2006).
} 
le font contrairement aux adultes (comme jouer à cache-cache, se jeter de l'eau, se salir en jouant ou s'asseoir par terre). Avec les femmes, je me conforme à la morale genrée et je respecte les règles corporelles qui régissent la séparation entre les hommes et les femmes (Sarcinelli, 2014) : je porte des jupes longues, je sers à table les hommes et mange après eux. Ou encore, pour sortir avec les jeunes filles au centre-ville, elles me prêtent leurs vêtements et me font me maquiller. En revanche, pour pouvoir rentrer en relation et avoir des conversations avec M. Tencovic, je ne fais pas la vaisselle, je mange avec les hommes et je me fais servir par les femmes. Enfin, avec les Gheorghe, je m'intègre au « corps de la famille » en tant que marraine : je partage les espaces de leur intimité, la nourriture qui leur est donnée par les enseignants et les vêtements qu'ils récupèrent.

Afin de franchir les frontières symboliques me séparant de mes interlocuteurs, j’ai dû également me démarquer des propos, des émotions et des sentiments moraux souvent exprimés par les acteurs non-roms : non seulement en évitant d'exprimer des jugements, mais aussi de faire transparaître certaines de mes émotions comme la peur de me rendre seule au camp, le dégoût et la préoccupation de partager des repas préparés en bidonville dans des conditions peu hygiéniques ou encore l'indignation face à certains de leurs comportements (notamment vis-à-vis des filles et des femmes). J'apprends rapidement la perception différente que mes interlocuteurs et moi avons d'une même situation, comme c'est le cas de l'odeur de déchets et produits chimiques provenant de l'usine située aux abords du camp (écœurante et insupportable pour moi, habituelle pour les enfants que je fréquente). Tout comme je perçois différemment les odeurs, mes réactions émotionnelles ne correspondent pas à celles de mes interlocuteurs. Par exemple, une fille de treize ans s'est moquée de moi en percevant ma crainte lors d'une descente de police. Si les "réflexes moraux » comme les sentiments d'indignation se présentent différemment selon les stades de l'enquête, d'autres émotions caractérisent le moment où l'on dépasse les frontières symboliques et morales qui séparent le chercheur de ses interlocuteurs (Bourgois, 2001), et où les normes morales du chercheur sont bousculées (Payet, 2011).

Néanmoins, mes efforts de ne pas exprimer mes réactions et jugements comme les autres non-Roms ne passent pas inaperçus. S’attendant à ce que je fasse œuvre de paternalisme, d'indiscrétion ou encore des reproches (par exemple qu'ils ne sont pas suffisamment attentifs à la scolarisation et à l'éducation des enfants), je suis au départ la cible de leurs stratégies d'évitement. Si l'observation de ces stratégies me permet de connaître des aspects cruciaux des relations que ces parents entretiennent avec la société locale, sur le long terme la relation d'enquête risquait de rester marquée par le pouvoir, l'inégalité et la violence typiques des rapports entre les deux mondes sociaux auxquels mes interlocuteurs et moi-même appartenons respectivement. Même si je n’ai pas pu échapper complètement aux dynamiques structurant les relations entre Roms et non-Roms, au fur et à mesure que je partageais le quotidien et les moments moins ordinaires des familles, je suis devenue parfois complice de leurs stratégies de 
résistance : j’aide par exemple une jeune mère à justifier aux autorités locales la situation de son enfant, en épurant les éléments peu présentables de son parcours.

Le fait que je n'exprime pas certaines de mes réactions n'implique pas, bien entendu, que je porte un regard distancié vis-à-vis des vies quotidiennes des familles marquées par des activités illégales, certains (rares) épisodes de maltraitance, ainsi que par un continuum d'événements dramatiques (comme des accidents, des agressions diverses et des menaces de la part des forces de l'ordre). Que du contraire! Ne pouvant renoncer ni à la nécessité de poser un regard scientifique ni à mes émotions, mes préjugés et mes sentiments moraux, il m'est apparu plus utile de les inclure dans le processus de connaissance (Girola, 1996). Ce travail d'articulation entre ces deux niveaux ne pouvait pas être conduit a posteriori en réintroduisant, au moment de l'analyse, une réflexivité et une " réflectivité » sur les données produites. Au contraire, ces deux niveaux ont été pris en compte au cours du processus d'enquête : c'est l'autoanalyse de mes propres réactions et le constat du décalage avec celles de mes interlocuteurs qui m'ont permis de prendre conscience des précautions nécessaires lors du travail de restitution. Enfin, au moment de l'analyse, la relation d'enquête s'est avérée révélatrice d'aspects qui régissent les relations de ces familles avec la société locale. En redonnant une place aux corps des interlocuteurs comme à celui de l'enquêteur, il est possible de situer dans l'expérience incorporée des sujets la genèse des processus historiques et culturels, y compris du processus de production d'un savoir anthropologique.

\section{Les tactiques de protection de l'intimité}

Malgré mon intégration, la relation d'enquête demeure précaire et mes interlocuteurs s'inquiètent des répercussions que mon travail pourrait avoir sur leur vie ${ }^{10}$. La suspicion, la réticence de mes interlocuteurs et le contrôle de l'image du groupe ne sont pas considérés seulement en tant que difficultés méthodologiques, mais aussi comme des données empiriques (Mauger, 1991). Or ces attitudes ont été souvent présentées comme des spécificités des Roms (Piasere, 1984 ; 1999), voire comme un véritable système de "protection de l'intimité » (Cossée, $2002:$ 49-50). Or, penser en ces termes me semble entraîner de forts risques de culturalisme et de réification de l'altérité (Abu Lughod, 2010). Les tentatives de me cacher quelque chose, surtout quand l'information est ensuite révélée, posent, certes, la question de l'ambiguïté de mon statut à mi-chemin entre le in-group et le out-group dont parle Cossée (2002), mais révèlent aussi la tentative de rééquilibrer et de subvertir le rapport de pouvoir, qui est au départ nettement en ma faveur. Ces dynamiques dévoilent les rapports sociaux de

${ }^{10}$ Une brève enquête sociologique a été menée par Giovanni Semi (Semi et al., 2007). 
domination et d'infériorisation que mes interlocuteurs vivent au jour le jour, mécanisme loin d'être culturel, mais que l'on retrouve chez bien d'autres situations minorités (Bourgois, 2001 ; Venkatesh, 2011).

À cet égard, les moments de négociation d'entretiens sont assez révélateurs. L'institutrice Marisa me présente à $M$. Tencovic, jeune père de trois enfants, né il y a une trentaine d'années en ex-Yougoslavie sans qu'aucun document ne puisse l'attester, de telle sorte qu'il ne peut même pas être déclaré comme apatride. M. Tencovic décline d'abord l'entretien sous prétexte qu'il ne sait pas si je vais livrer les informations à la police, puis se déclare prêt à me l'accorder à la condition que je fasse un mariage blanc avec lui ou que je le paie. Il finit par me donner rendez-vous le lundi suivant pour un entretien non enregistré. Le jour venu, la rue du camp est particulièrement déserte et sa maison a l'air vide. Sa femme m'annonce que son mari n'est pas là et que, de toute façon, il ne veut pas se faire interviewer. Elle m'explique que le matin son mari a failli se faire arrêter lors d'une descente de police sur le terrain, il a dû s'échapper par la fenêtre et il n'est pas rentré depuis. Elle m'invite toutefois à rester et me prépare le café. À son retour, M. Tencovic déclare qu'il ne va plus se faire interviewer. Sans nationalité ou statut d'apatride, il n'a aucune véritable alternative entre « assumer une certaine déviance » (Coutant, 2001 : 34) et me faire connaître le monde de marge auquel il appartient, ou bien refuser l'enquête (Mauger, 1991). Quand sa tante arrive à la maison, il me présente comme «la juge », comme si j'étais une professionnelle liée au monde judiciaire, un terme qu'il utilisera à plusieurs reprises et qui ne laisse aucun doute sur la crédibilité qu'il accorde à mon identité de chercheuse. Je ne ferai jamais d'entretien, mais les Tencovic acceptent la méthode de l'observation participante et de l'entretien informel sur le mode de la conversation ordinaire, devenant parmi mes principaux informateurs. Par la suite, M. Tencovic me propose d'interviewer sa voisine, à condition de le faire chez lui et qu'il assiste à l'entretien. N'étant pas parmi les hommes les plus influents au sein du campement (que du contraire !), il cherche ainsi à rééquilibrer le rapport de pouvoir, qui n'est pas en sa faveur. Il érige sa voisine en «porte-parole » (Mauger, 1991 : 135-136), citoyenne italienne depuis longtemps, employée durant une période de sa vie et domiciliée par moments dans des camps autorisés. Reste que cette situation n'est pas sans conflit: durant l'entretien, $\mathrm{M}$. Tencovic nous interrompt à une ou deux reprises pour accuser sa voisine de révéler plus que ce qu'elle n'est censée faire, les deux ayant des positions différentes au sujet du contrôle de l'image du groupe vis-à-vis de l'extérieur.

Toutefois, la question qui se pose dans bien des cas n'est pas tant de cacher des tabous afin de garder une frontière vis-à-vis de l'extérieur que de savoir comment et dans quelle mesure assumer sa propre déviance face au chercheur. Quand j'explique ma démarche à un autre couple du réseau des familles en insistant sur l'anonymat, M. Markovic déclare qu'ils n'ont rien à cacher tandis que sa femme demande aussitôt à l'institutrice Marisa: «De toute façon, elle le sait, n'est-ce pas ?»; "Oui, elle sait tout » répond-t-elle. Par «le », Mme G. Markovic résume tout ce qui n’est pas conforme 
aux normes ambiantes, notamment leurs pratiques illégales. Dans la mesure où je le sais, l'assumer est la meilleure option suivant aussi ce que Coutant (2001) appelle une «logique d'optimisation de la conformité » (p. 33). Or les choses se passent différemment dans la famille Brenovic dont les membres, ayant les "propriétés objectives » et 《subjectives $~^{11}$ pour apparaître conforme, ont du mal à assumer leurs comportements déviants (comme par exemple leurs activités illégales) vis-à-vis de moi.

En raison de ces comportements «peu conformes » voire carrément illégaux, je me suis également retrouvée face à des informations inexactes, mensongères, voire inventées de toutes pièces. Il arrive que $\mathrm{M}$. Tencovic me dise quelque chose et, une fois parti, sa femme avoue «la vérité », me priant de ne pas le dire à son mari, ou encore que des enfants me révèlent des informations qu'on leur a interdit de partager. D'une part, je procède à une " herméneutique de la méfiance », la méthode proposée par Alba Zaluar (2004:12) qui consiste à comparer et vérifier systématiquement les énoncés des interlocuteurs avec d'autres sources d'informations. D'autre part, à l'instar de Zaluar, je m'interroge sur les significations des mensonges systématiques, examinant le sens des contrevérités recueillies auprès de chaque membre des familles à différentes occasions. Dans un premier stade du terrain, le mensonge est un instrument pour tester ma naïveté. Ensuite, chaque acteur s'autorise à me dire certaines choses selon le moment et les personnes qui nous entourent. Enfin, je suis moi-même sollicitée pour participer à la construction d'un mensonge comme lorsque j'accompagne une mère au bureau d'État civil me demandant de mettre au point ce que nous allons dire au fonctionnaire du bureau pour cacher que son mari est en prison. Ce déplacement de mon rôle de destinataire du mensonge à complice me permet de mieux en saisir la fonction : il s'agit, en l'occurrence, d'esquiver le stigmate d'être la femme d'un détenu. Si les scènes ethnographiques où intervient le mensonge sont replacées dans un réseau de relations intersubjectives, les mensonges dont je suis la "victime» comme ceux dont je suis complice acquièrent une signification. Il s'agit des tactiques (de Certeau, 1990) pour écarter des assignations explicites ou implicites, comme notamment celle d'être des « mauvais parents ».

Un autre subterfuge pour maîtriser la relation d'enquête est de recourir à l'humour (Griddiths, 2002 ; Madec, 2002) : « l'humour, de contrainte, devient une ressource de la relation d'enquête agissant comme révélateur de rapports de pouvoir entre enquêtrice et enquêtés » (Mainsant, 2008: 102). Quand j'arrive chez M. Tencovic pour l'interviewer, il dit en riant qu'il sent qu'il va avoir un infarctus. Ensuite, il me pro-

\footnotetext{
11 «Des propriétés objectives (le fait de vivre en famille, de disposer d'un minimum de ressources licites; la compétence nécessaire pour accomplir une démarche administrative ; la nationalité du conjoint, etc.) et des propriétés subjectives comme la capacité à maîtriser sa propre image » (Coutant, $2001: 3$ ).
} 
pose d'aller trois jours en France avec lui pour que je loue une voiture à mon nom. Et quand je lui dis que je n'ai pas le permis, il m'en offre un. Puis, il plaisante sur le fait qu'il pourrait tomber sur mon appartement quand il vole. N'estimant pas pouvoir susciter ni mon estime ni ma pitié, $M$. Tencovic préfère m'étonner face à l'inconfort d'une relation d'enquête vécue comme un "quasi-procès " (Mauger, 1991: 137). Ses plaisanteries peuvent être interprétées comme des «lieux du discours et des interactions qui cristallisent d'ambivalents processus de stigmatisation et de négociation du stigmate» (p. 116). Quand M. Tencovic évoque la possibilité de cambrioler mon appartement à son insu, il me rappelle que j'appartiens aux « autres », ceux chez qui il va voler. Pour être de « leur côté » à eux, je dois participer aux activités illégales : avec cette invitation, renouvelée au cours de l'enquête, les membres de la famille "plaisantent à l'idée de briser une vocation de sociologue et visent à créer l'illusion de la conversion du chercheur, de son passage du côté de ses enquêtés» (p.111). Au fil de ces interactions prenant la forme d'un jeu auquel je participe se crée une sorte de confiance avec mes interlocuteurs. En définitive, si l'on aborde la suspicion, le contrôle de l'image du groupe, les mensonges, la réticence à parler ou encore l'humour comme des données et non pas comme des obstacles, ils nous apportent une compréhension plus fine de l'expérience de vie de nos interlocuteurs.

\section{Conclusion}

À titre de conclusion d'une réflexion sur l'engagement du corps et des émotions de l'ethnographe d'un point de vue théorique, méthodologique et épistémologique, il importe de revenir sur l'introduction à l'ouvrage pionnier de Georges Devereux (2003 [1980]), où Weston La Barre illustre comment le concept de « contre-transfert » permet à Devereux d'utiliser ses propres émotions en tant que moyen de rendre intelligible ce qui fait obstacle à sa compréhension de la réalité examinée. Or, comme le défend Andrew Beatty (2010), il n'est pas possible de faire confiance et de compter sur nos propres émotions pour comprendre celles de personnes très différentes de nous. Prendre au sérieux l'expérience corporelle et émotionnelle de l'ethnographe et pratiquer une auto-analyse signifie alors descendre de la tour d'ivoire et considérer «l'ethnologue à la fois comme sapiens, comme porteur d'une certaine culture » et rendre compte de «comment l'observateur de faits humains réagit en tant que personne et en tant qu'être humain à ses propres observations » (La Barre, 2003 [1980] : 7). Bien que modeste, ma tentative s'est voulue à la fois un acte de transparence, permettant de questionner et de problématiser l'expérience de terrain normalement tenue pour acquise, et une manière de restituer la rencontre ethnographique dans son épaisseur de scène historique de la rencontre recherchée par le chercheur et acceptée par l'interlocuteur en tant qu'expérience corporelle et émotionnelle.

Si un certain nombre des émotions évoquées dans ces pages sont similaires à celles décrites par Bronislaw Malinowski (1963 [1922]) (le sentiment d'altérité, la solitude, 
l'inconfort, l'absence de familiarité) auxquels je réponds par un processus de domestication et de rationalisation (Benson, O'Neill, 2007), d'autres (l'insécurité, la peur du harcèlement sexuel, la méfiance, le bouleversement et la suspicion) laissent transparaître des aspects propres aux terrains caractérisés par une distance sociale importante entre le chercheur et ses interlocuteurs, comme entre les pratiques et les représentations de l'un et des autres (Duvoux, 2014). Tout comme la suspicion, la réticence de mes interlocuteurs et le contrôle de l'image du groupe, aussi l'inquiétude du chercheur a été pensée moins en tant qu'obstacle que comme "une condition de l'intelligibilité anthropologique et sociologique des sociétés humaines » (Fassin, 2008 : 10). Face à des terrains particulièrement sensibles, la nécessité d'être attentif à ses propres réactions est d'autant plus fondamentale (Devereux, 2003 [1980]), car des sentiments humanitaires et moraux s'éveillent en nous et il arrive facilement de se retrouver davantage lié « émotionnellement et moralement » à son propre objet (Fassin, 2008) - comme cela ressort de mon propre cahier de terrain.

Bien que je ne puisse pas «parvenir à objectiver complètement les relations sociales, ni à éviter tout jugement de valeur » (Fassin, 1998 : 42), la prise en compte, tout au long du travail de recherche, des éléments ici esquissés, amène à examiner une série de dimensions objectives et subjectives du travail d'enquête (comme les émotions, les ambiguïtés, les tensions) et à mieux saisir l'ensemble des conditions qui marquent une expérience de terrain, dans ce cas en l'occurrence la spécificité d'être "chez moi » et pourtant «étrangère ». Le recours à une sorte de biographie ethnographique devient alors révélateur des enjeux scientifiques, sociaux et éthiques inhérents à une enquête donnée. Les éléments qui en ressortent pourraient sembler anecdotiques, mais ils laissent apparaître une vérité sur les expériences du chercheur et des interlocuteurs. Ils sont à ce titre utiles pour mieux saisir ce qui se joue non seulement dans ces expériences de vie, mais aussi dans les conditions de possibilités de ces existences qu'on risquerait de négliger si on s'en tenait au niveau de l'herméneutique discursive ou textuelle.

\section{Bibliographie}

ABu-Lughod L. (2010), "Écrire contre la culture. Réflexions à partir d'une anthropologie de l'entre-deux », in D. CEFAÏ (dir.), L'engagement ethnographique, Paris, Éditions de l'École des hautes études en sciences sociales («En temps et lieux »), p. 417-445.

BeATTY A. (2010), «How did it feel for you? Emotion, narrative, and the limits of ethnography », American Anthropologist, vol. 112, n 3, p. 430-443.

BENSON P., O’Neill K. L. (2007), "Facing risk: Levinas, ethnography and ethics», Anthropology of consciousness, vol. 18, $n^{\circ} 2$, p. 29-55. 
BORDIGONI M. (2001), «"Terrain désigné”, observation sous contrôle : quelques enjeux d'une ethnographie des Tsiganes », Ethnologie française, vol. 31, n 1, p. 117-126.

Bourgois P. (2001), En quête de respect. Le crack à New York, Paris, Seuil.

BRUNER E. M. (1986), «Experience and its expression », in V. W. TURNER, E. M. BRUNER, The anthropology of experience, Urbana, University of Illinois Press, p. 3-32.

Cefaï D. (2010), L'engagement ethnographique, Paris, Éditions de l'École des hautes études en sciences sociales (" En temps et lieux»).

CLIFFoRD J. (1996 [1988]), «De l'autorité en ethnographie », in Malaise dans la culture: l'ethnographie, la littérature et l'art au XX' siècle, Paris, Éditions de l'École nationale supérieure des Beaux-Arts.

Clifford J., Marcus G. (dir.) (1986), Writing culture: the poetics and politics of ethnography, Berkeley, University of California Press.

COSSEE C. (2002), « Familles tsiganes et protection de l'intimité », Ethnologie Française, vol. 32, $\mathrm{n}^{\circ} 1, \mathrm{p} .49-59$.

Coutant I. (2001), "Statu quo autour d'un squat», Actes de la recherche en sciences sociales, vol. 136-137, $\mathrm{n}^{\circ} 1-2$, p. 27-37.

CsORDAS T. J. (1999), "Embodiment and cultural phenomenology", in G. WEISS, H. F. HABER (dir.), Perspectives on embodiment: the intersections of nature and culture, New York, Routledge, p. 143-162.

Certeau M. de (1990), L'invention du quotidien. 1. Arts de faire, Paris, Gallimard.

DEVEREUX G. (2003 [1980]), De l'angoisse à la méthode dans les sciences du comportement, Paris, Aubier.

Duvoux N. (2014), « La peur de l'ethnographe. Réflexions à partir d'une enquête sur la pauvreté urbaine à Boston », Genèses, vol. 4, n 97, p. 126-139.

FASSIN D. (1998), "L'anthropologie entre engagement et distanciation. Essai de sociologie des recherches en sciences sociales sur le sida en Afrique», in C. BeCKeR, J.-P. Dozon, C. Овво, M. Toure (dir.), Vivre et penser le sida en Afrique, Paris, IRD/Karthala/Codesria, p. 41-66.

FASSIN D. (2008), «Introduction. L’inquiétude ethnographique », in D. FASSIN, A. BENSA (dir.), Les politiques de l'enquête. Épreuves ethnographiques, Paris, La Découverte, p. 7-15.

FASSIN D., BENSA A. (dir.) (2008), Les politiques de l'enquête. Épreuves ethnographiques, Paris, La Découverte. 
FASSIN D., FASSIN É. (dir.) (2006), De la question sociale à la question raciale? Représenter la société française, Paris, La Découverte.

FAVRET-SAADA J., CONTRERAS J. (1981), Corps pour corps. Enquête sur la sorcellerie dans le bocage, Paris, Gallimard.

FouRNIER P. (2006), «Le sexe et l'âge de l'ethnographe: éclairants pour l'enquêté, contraignants pour l'enquêteur », Ethnographiques.org, vol. 11.

GiRola C. (1996), "Une anthropologie réflexive: la rencontre avec les sans-abri », Politix, vol. 34, p. 87-98.

GRIDDITHS L. (2002), «Humor as resistance to professional dominance in community mental health teams », in S. TAYLOR (dir.), Ethnographic research. A reader, Londres, Sage, p. 213-233.

JACKSON M. (1983), « Knowledge of the body », Man, vol. 18, p. 327-345.

LA BARRE W. (2003 [1980]), « Préface » in G. DevereuX, De l'angoisse à la méthode dans les sciences du comportement, Paris, Aubier, p. 5-9.

MADEC A. (2002), « Rire et relations d'enquête », Ethnologie française, vol. 1, n 32, p. 8994.

MAINSANT G. (2008), « Prendre le rire au sérieux. La plaisanterie en milieu policier » in D. FASSIN, A. BENSA (dir.), Les politiques de l'enquête. Épreuves ethnographiques, Paris, La Découverte, p. 99-120.

Malinowski B. (1963 [1922]), Les Argonautes du Pacifique occidental, Paris, Gallimard.

MASCARENHAS-KeYES S. (1987), "The native anthropologist: constraints and strategies in research", in A. JACKSON (dir.), Anthropology at Home, Londres, Tavistock, p. 180-195.

MAUGER G. (1991), « Enquêter en milieu populaire », Genèses, vol. 6, p. 125-143.

OKELY J. (1992), «Anthropology and autobiography. Participatory experience and embodied knowledge », in J. OKelY, H. Callaway (dir.), Anthropology and autobiography, Londres, New York, Routledge, p. 1-28.

OkelY J. (1984), « Fieldwork in the Home Countries », Rain, vol. 61, p. 4-6.

PACK S. (2006), « Social thought \& commentary: how they see me vs. how I see them: the ethnographic self and the personal self », Anthropological Quarterly, vol. 79, $\mathrm{n}^{\circ}$ 1, p. 105-122.

PAYET J. P. (2011), « L'enquête sociologique et les acteurs faibles », SociologieS.

Peirano M. (1998), « When anthropology is at home: the different contexts of a single discipline », Annual Review of Anthropology, vol. 27, p. 105-128. 
PIASERE L. (1984), Mare Roma. Catégories humaines et structure sociale. Une contribution à l'ethnologie tsigane, thèse de doctorat, Paris, École des hautes études en sciences sociales.

PIASERE L. (1999), Un mondo di mondi. Antropologia delle culture rom, Naples, L'ancora.

PiASERE L. (2006), « Che cos'é un campo nomadi ? », Achab Rivista di Antropologia, vol. 8, $n^{\circ}$ 6, p. 8-16.

SARCinElli A.S. (2007), "O outro en se mesmo. Etno-antropologia del pensiero brasiliano », Quaderni di Thule, Rivista italiana di studi americanistici, vol. 7, p. 311320.

Sarcinelli A.S. (2014), Protéger, éduquer, exclure. Anthropologie de l'enfance et de la parentalité roms en Italie, thèse de doctorat, Paris, École des hautes études en sciences sociales.

SARCinelli A. S. (2015), «Ordres et désordres des âges dans les familles transnationales roms », Ethnologie française, vol. 4, n 154, p. 705-714.

SARCINELLi A. S. (2016), « Les lois de l’habitat précaire dans les campi rom non autorisés en Italie. Action publique et militantisme entre reconnaissance et méconnaissance », in G. Cousin, G. Loiseau, L. Viala, D. Crozat, M. LiÈvre (dir.), Actualité de l'Habitat Temporaire. De l'habitat rêvé à l'habitat contraint, Marseille, Terra HN Éditions ( SHS »).

SCHEPER-HughES N. (1992), Death without weeping: the violence of everyday life in Brazil, Berkeley, University of California Press.

SEMI G., Cicuto A., CoRRADI A. (2007), « Il villaggio e il quartiere : il campo di via Monte Bisbino», in M. Ambrosins, A. Tosi (dir.), Vivere ai margini. Un'indagine sugli insediamenti rom e sinti in Lombardia. Osservatorio Regionale per l'integrazione e la multi etnicità, Rapporto 2006, Milan, Fondazione Ismu, p. 101-129.

TURnER A. (2000), "Embodied ethnography. Doing culture», Social Anthropology, vol. 8, p. 51-60.

WEBER F., NOIRIEL G. (1990), « Journal de terrain, journal de recherche et auto-analyse. Entretien avec Florence Weber », Genèses, vol. 2, p. 138-147.

Venkatesh S. (2011), Dans la peau d'un chef de gang, Paris, L'école des loisirs.

Zaluar A. (2004), Integração perversa: probreza e tráfico de drogas, Rio de Janeiro, Editora FGV. 